\title{
黄土高原腹地人工林下草本层群落构建机制
}

\author{
施晶晶 1,2 赵鸣飞 1,2 王宇航 1,2 薛 峰 ${ }^{1,2}$ 康慕谊 3 , 江 源 $1,2,3 *$
}

${ }^{1}$ 北京师范大学地理科学学部自然资源学院, 北京 $100875 ;{ }^{2}$ 北京师范大学地理科学学部中药资源保护与利用北京市重点实验室, 北京 $100875 ;{ }^{3}$ 北京 师范大学地表过程与资源生态国家重点实验室, 北京 100875

摘 要 群落构建机制是生态学研究的核心论题。生态位理论和中性理论是阐明群落构建的两种主要理论, 但这两种理论分 别强调的环境过滤和扩散限制对群落构建的影响尚存争议。该研究以黄土高原人工林下草本层群落为研究对象, 将群落物种 组成数据与地理空间、气候、地形以及生物因子相结合, 运用随机森林模型(RF)和基于距离矩阵的多元回归方法(MRM), 探 究了各个因子的重要性, 并通过邻体主坐标矩阵(PCNM)和基于距离的咒余分析(db-RDA)分别篎选显著的空间结构和环境因 子, 最后结合篎选出的PCNM特征值和环境变量进行变差分解。研究结果显示: 1)林下草本层群落组成相似性随地理距离和生 境差异的增加而减小。2)地理距离与生境差异共同解释了群落组成相似性变化的 $47.8 \%$ 。其中, 空间因子和环境因子的独立 解释率分别为 $14.1 \%$ 和 $9.8 \%$, 两者的联合解释率为 $23.9 \%$ 。 3 )地理距离和年降水量是引起研究区内物种组成变化的关键因子, 且地理距离的重要性大于年降水量。在黄土高原腹地, 扩散限制与环境过滤共同主导了人工林下草本层群落的构建过程。

关键词＼cjkstart群落构建; 生态位理论; 中性理论; 环境过滤; 扩散限制; $\beta$ 多样性; 林下草本层群落

施晶晶, 赵鸣飞, 王宇航, 薛峰, 康慕谊, 江源 (2019). 黄土高原腹地人工林下草本层群落构建机制. 植物生态学报, 43, 834-842. DOI: $10.17521 /$ cjpe.2018.0153

\section{Community assembly of herbaceous layer of the planted forests in the central Loess Plateau, China}

SHI Jing-Jing ${ }^{1,2}$, ZHAO Ming-Fei ${ }^{1,2}$, WANG Yu-Hang ${ }^{1,2}$, XUE Feng ${ }^{1,2}$, KANG Mu-Yi ${ }^{3,1}$, and JIANG Yuan ${ }^{1,2,3^{*}}$

${ }^{1}$ College of Resources Science \& Technology, Faculty of Geographical Science, Beijing Normal University, Beijing 100875, China, ${ }^{2}$ Beijing Key Laboratory of Chinese Medicine Resource for Protection and Utilization, Faculty of Geographical Science, Beijing Normal University, Beijing 100875, China; ${ }^{3}$ State Key Laboratory of Earth Surface Processes and Resource Ecology, Beijing Normal University, Beijing 100875, China

\section{Abstract}

Aims Many competing theories seek to identify the mechanisms behind the community assembly. Niche theory and neutral theory are among the two most competing mechanisms. Exploring factors controlling the patterns of $\beta$-diversity is an approach to reveal the underlying mechanisms of community assembly. In this paper, we aim to reveal the $\beta$-diversity pattern of understory in planted forests of the central Loess Plateau and to evaluate the relative influences of deterministic (environmental filtering) and stochastic (dispersal limitation) processes on the community assembly.

Methods We sampled in total 107 sites of planted forests spatially spreading over the central Loess Plateau. In each site, three plots $(10 \mathrm{~m} \times 10 \mathrm{~m})$ were randomly designed each at a distance of about $50 \mathrm{~m}$. The floristic composition, community structure, and environmental conditions were investigated in each plot. We calculated the dissimilarity $\left(\beta_{\mathrm{sim}}\right)$ of species composition, geographic distance and environmental divergence for each of the 5671 site pairs. We used random forest (RF) to quantify the influence of the selected factors on species composition, and distance-based multivariate regression method (MRM) to fit the relationship between dissimilarity, geographic distance and environmental divergence. To quantify how and in what extent the environmental filtering and the dispersal limitation influence the species composition among sites, we performed the principle coordinates of neighbor matrices (PCNM) and distance-based RDA (db-RDA) to select the pronounced independent variables. We then partitioned the variance into three parts, namely, 1) independent contributions of geographic distance; 2) independent contributions of environmental divergence; and 3) the joint contribution of both.

Important findings The community similarity declined with increasing geographical distance and environmental divergence. Geographical distance and environmental divergence together explained $47.8 \%$ of variance in

收稿日期Received: 2018-07-03 接受日期Accepted: 2018-10-18

基金项目: 国家科技基础性工作专项(2011FY110300)和国家自然科学基金(41630750和41271059)。Supported by the National Basic Work of Science and Technology of China (2011FY110300), and the National Natural Science Foundation of China (41630750 and 41271059).

* 通信作者Corresponding author (jiangy@bnu.edu.cn) 
the dissimilarity in species composition. The spatial variables accounted for $14.1 \%$, while the environmental variables accounted for another $9.8 \%$ of the variance in dissimilarity of species composition. Annual precipitation and basal area of trees are the two most important environmental factors influencing the pattern of dissimilarity of species composition. These results implied that both environmental filtering and the dispersal limitation play important roles in shaping the community assembly of the herbaceous layers of planted forests in the central Loess Plateau.

Key words community assembly; niche theory; neutral theory; environmental filtering; dispersal limitation; $\beta$ diversity; understory community

Shi JJ, Zhao MF, Wang YH, Xue F, Kang MY, Jiang Y (2019). Community assembly of herbaceous layer of the planted forests in the central Loess Plateau, China. Chinese Journal of Plant Ecology, 43, 834-842. DOI: 10.17521/cjpe.2018.0153

揭示群落物种空间分布格局和动态变化机制即 群落构建机制是群落生态学的基本目标(Smith \& Lundholm, 2010; May et al., 2015; Gibson et al., 2017)。生态位理论(确定过程)和中性理论(随机过程) 是阐明该机制的两种主要理论(Page \& Shanker, 2018), 但这两个理论对群落构建过程的相对重要 性目前仍未达成广泛共识 (Leibold \& McPeek, 2006)。生态位理论认为物种组成差异格局是环境异 质性的产物, 共存物种间的生态位分化、资源竞争 等确定性因素是主要决定因子(Chase \& Myers, 2011)。然而, 中性理论则认为群落中相同营养级所 有个体在生态上等价, 差异主要由扩散限制和生态 漂变等随机作用主导(Hubbell, 2001)。近年来的研究 则倾向于整合两个理论的观点, 认为确定过程和随 机过程均在群落构建过程中发挥着重要作用 (Gravel et al., 2006; Adler et al., 2007; Chase \& Myers, 2011)。

$\beta$ 多样性格局反映群落间物种组成的变化情况 (Anderson et al., 2011; Legendre \& de Cáceres, 2013), 揭示 $\beta$ 多样性格局与空间距离和环境异质性之间的 关系可衡量生态位理论和中性理论对群落构建过程 的相对重要性(Condit et al., 2002; Kraft et al., 2011)。 若 $\beta$ 多样性格局主要受环境过滤影响, 则环境变量 对样地间物种组成变化的解释率将最高(Petermann et al., 2015; Jamoneau et al., 2018)。若中性理论占主 导地位, 则扩散限制是 $\beta$ 多样性格局的主要影响因 素, 植物种子的拓殖速率与地理距离之间将呈负相 关关系。换言之, 地理距离或者空间属性(如采样粒 度、研究范围等)将在群落构建过程中扮演更为重要 的角色(Seidler \& Plotkin, 2006; Page \& Shanker, 2018)。

量化确定性和随机过程对群落构建的相对重要 性，可综合考虑空间因子、环境条件以及物种本身
的功能属性差异等因素(Condit \& Hubbell, 2002), 通过变差分解法分解群落间 $\beta$ 多样性的差异进行分 析(Legendre et al., 2009; Burrascano et al., 2011; Myers et al., 2013)。Gibson等(2017)在澳大利亚西南 部沙地平原的研究中指出, 模型所选变量(气候、土 壤和空间变量)共可解释群落物种组成变化的 $87 \%$, 变差分解的结果表明空间和气候因子解释了绝大部 分变化。除了变差分解, 相似性随距离的衰减斜率 也是研究群落构建机制的常用方法(Anderson et al., 2011; Siefert et al., 2013)。Tang等(2013)在中国北方 主要草原群落的研究表明，控制环境“空间自相关” 的影响后, 群落物种组成相似性随地理距离和环境 差异的增大而减小。

黄土高原地区是我国当前主要造林并大量栽植 抚育人工林的区域(Fu et al., 2011)。相比于生物热点 区, 在环境条件相对恶劣的黄土高原进行群落构建 机制的研究相对较少(王丹等, 2013)。该地区现有的 经验案例显示, 确定性和随机性过程对群落构建的 作用受不同研究对象和研究尺度的影响。王丹等 (2013) 以黄土高原子午岭地区的草地植物群落为对 象, 研究表明确定性过程和随机过程均在群落构建 过程中发挥了作用, 但随机过程扮演了更为重要的 角色。而王世雄等(2016)对黄土高原油松(Pinus tabuliformis)林群落异质性进行分析后得出, 生境异 质性与群落异质性呈现极显著的线性关系, 即认为 生态位理论是该区群落构建的主导机制。但已有研 究多为集中在某一生境(如马栏林区)的小尺度研究, 对于区域尺度的关注稍显不足。而确定性过程和随 机过程对物种组成变化的相对重要性具有尺度效应 (Chase, 2014), 并且不同植被类型对于环境和空间 因子的响应不同(Tuomisto et al., 2017)。因此, 有 必要在区域尺度上探索黄土高原地区的群落构建 过程。 
林下草本层是森林生态系统的重要组成部分 (Gilliam, 2007), 在森林生态系统中往往占有最高比 例的物种多样性(占据森林物种丰富度的 $90 \%$ 以上) (Gilliam et al., 2006), 其不仅能促进森林生态系统 养分循环, 稳定林分生产力(Sabatini et al., 2014; Márialigeti et al., 2016), 还能通过与乔木幼苗之间 的资源竞争影响乔木种苗的存活、发育与生长 (George \& Bazzaz, 2014)。然而, 相比于乔木层物种 多样性, 草本层物种多样性尚未引起广泛关注(尤 业明等, 2016)。鉴于黄土高原的特殊性以及林下草 本层的重要性, 本研究拟探究以下两个科学问题: (1)黄土高原地区人工林下草本群落 $\beta$ 多样性呈怎样 的格局? (2)环境过滤和扩散限制对人工林下草本 群落构建的相对重要性如何?

\section{1 材料和方法}

\section{1 研究区概况}

地处黄土高原腹地的研究区 $\left(34.47^{\circ}-39.32^{\circ} \mathrm{N}\right.$, $\left.107.57^{\circ}-111.53^{\circ} \mathrm{E}\right)$, 面积约 $1.0 \times 10^{5} \mathrm{~km}^{2}$, 最高海拔 约 $2000 \mathrm{~m}$, 最低海拔约 $800 \mathrm{~m}$ 。该区气候具有典型 的温带大陆性季风气候特征, 年平均气温3.6$14.3{ }^{\circ} \mathrm{C}$, 年降水量 150-750 mm, 且降水主要发生在 6-9月。降水最丰富的东南部地区年降水量为600$750 \mathrm{~mm}$, 而西部和西北部仅150-250 $\mathrm{mm}$, 呈现出
从东南向西北显著降低的水分梯度。研究区由暖温 带向温带过渡, 植被带跨越了落叶阔叶林带、森林 草原带和草原带等(孙龙等, 2016)。

本区域因黄土的自然属性以及历史上强烈的人 类活动导致水土流失严重，因而自20世纪60-70年 代起，广泛开展了人工栽植或飞机播种结合人工抚 育管理的造林工程(Xiao, 2014)，造林栽植的树种依 局地生境分异特征主要有: 油松、刺槐(Robinia pseudoacacia)、小叶杨(Populus simonii)等。其林下 原生灌木主要有沙棘(Hippophae rhamnoides)、柠条 锦鸡儿(Caragana korshinskii)、黄刺玫(Rosa xanthina) 等; 成林后的林下, 草本层主要有臭草 (Melica scabrosa)、赖草(Leymus secalinus)、长芒草(Stipa bungeana)、蒿类(Artemisia spp.)等。

\section{2 样地设置及数据预处理}

于2014-2016年7-8月, 采用样地系统取样方法, 在研究区沿水分梯度布设样地 107 个(图1)。其中79 个为刺槐林, 19 个为小叶杨林, 9 个为山杨林。在每个 样地内每隔50 m设置 3 个 $10 \mathrm{~m} \times 10 \mathrm{~m}$ 的样方。记录 样方内胸径 $\geqslant 3 \mathrm{~cm}$ 的乔木种名、胸径、高度及枝下 高, 估算乔木层盖度。利用胸径计算每木胸高断面 积, 按样方累加后得到胸高断面积和; 通过计算每 木树高与枝下高的平均差估算样地的冠层厚度。在 样方四角设置 $1 \mathrm{~m} \times 1 \mathrm{~m}$ 草本层样方, 记录其内物种

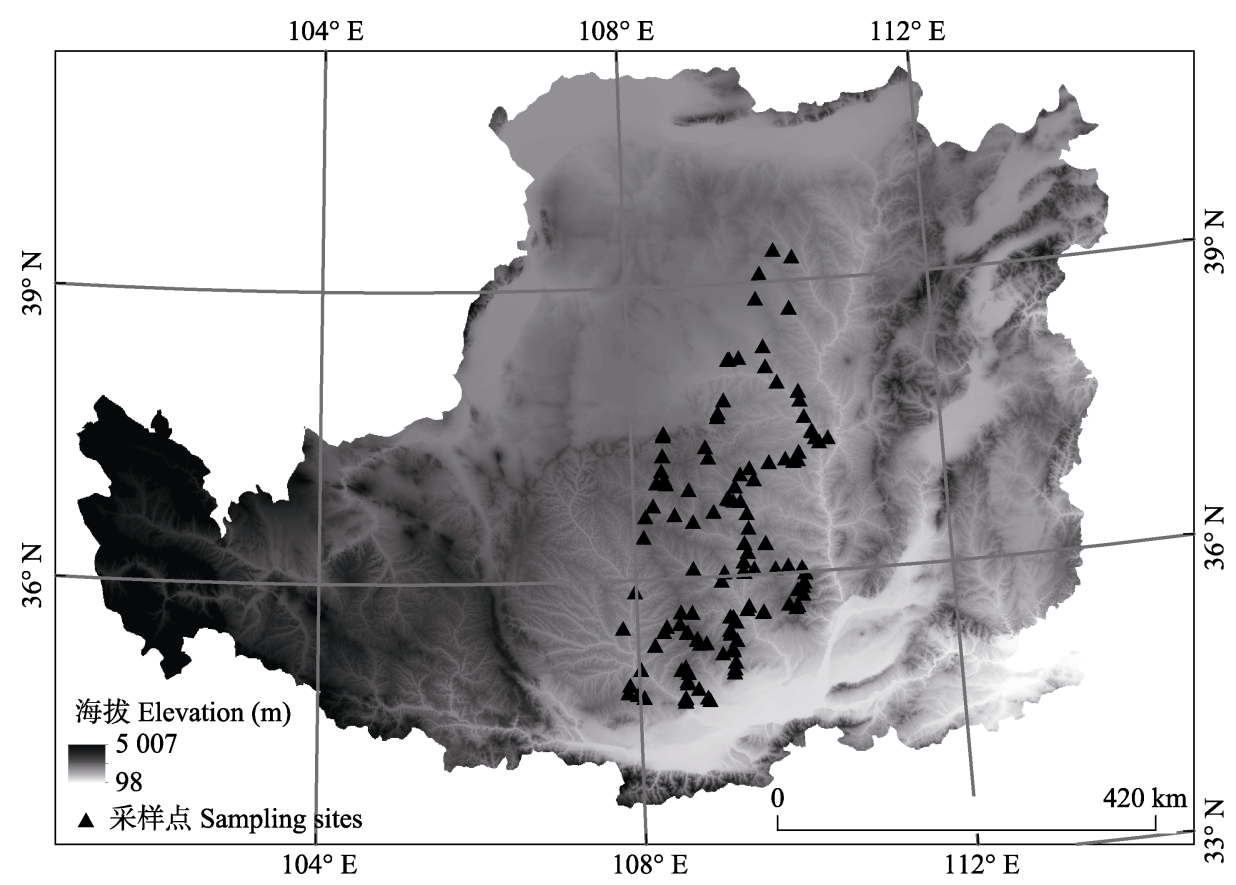

图1 黄土高原研究区概况及调查样地分布。

Fig. 1 Sketch map for the central Loess Plateau, showing the location for 107 sampling sites.

www.plant-ecology.com 
名称、盖度和高度。采用手持GPS仪记录样地的地 理信息(经纬度、海拔), 使用罗盘测量坡度、坡向。 最后, 将 3 个样方各个变量取均值用于后续的样地 数据分析。

将 $0^{\circ}-360^{\circ}$ 的方位角按照余弦公式转化为 $0-1$ 的 坡向指数 TRASP, 以反映生境的干湿状况, TRASP 值越大表示生境越干热(刘秋锋等, 2006), 计算公式 如下:

$$
\text { TRASP }=\frac{1-\cos ((\pi / 180)(\text { aspect }-30))}{2}
$$

式中, $T R A S P$ 为转换后的坡向指数, aspect为坡向的 角度数值。

气候数据来源于WorldClim数据库 $(2.0$ 版, http:// www.worldclim.org/, 分辨率 $1 \mathrm{~km} \times 1 \mathrm{~km}$ )。利用 ArcGIS10.2软件, 提取黄土高原地区各样地相应经 纬度坐标处的年平均气温和年降水量数据。

\section{3 数据分析}

\subsection{1 陊样性与距离矩阵计算}

采用目前衡量 $\beta$ 多样性常用的相似性或相异性 指数度量群落物种组成变化, 本研究选取基于有无二元数据的Simpson相异指数 $\left(\beta_{\text {sim }}\right)$ 计算配对样地 间物种组成的差异(Baselga, 2010), 具体公式如下:

$$
\beta_{\text {sim }}=\min (b, c) /[(\min (b, c)+a)]
$$

其中, $b$ 和 $c$ 为两样地的独有种, $a$ 为两样地共有种。 $\beta_{\text {sim }}$ 的范围为 $0-1$, 分别代表两配对样地间物种组成 的最大相似度和最小相似度。

计算107个样地所有配对样地即5 671对样地间 物种组成变化, 在等距离投影下基于各样方的经纬 度计算得到样地间地理距离矩阵。为了度量群落间 生境异质性的大小, 本研究选取气候因子(年平均 气温、年降水量)、生物因子(胸高断面积和、乔木 高、冠层厚度、乔木层盖度、草本层盖度及草本层 高度)以及生境因子(海拔、坡度和坡向)等构建环境 距离矩阵。为消除变量间因量纲差异引起的环境分 异对测量结果造成的潜在影响, 将上述环境变量进 行标准化 (平均值为 0 , 标准差为 1 ), 然后将该 11 个 变量分别转换为欧式距离矩阵。样地间物种组成变 化作为响应变量矩阵, 地理距离矩阵和环境距离矩 阵作为解释变量矩阵。

\subsection{2 统计分析}

运用随机森林模型(RF)评估上述各因子对群落 物种组成的相对重要性(以节点纯度的增加表示)。
随机森林模型除了可以在一定程度上忽略解释变量 间的空间自相关问题(Marmion et al., 2009), 还可以 处理群落物种组成与解释变量的非线性关系 (Breiman, 2001)。由于群落 $\beta$ 多样性的样对数据之间 不独立, 采用置换检验(999次)对基于距离矩阵的多 元回归(MRM)(Legendre et al., 1994)系数进行检验。 再由基于距离的冗余分析法(db-RDA)(Legendre \& Anderson, 1999), 用向前选择筛选出显著的环境变 量。通过邻体主坐标矩阵 $(\mathrm{PCNM})$ (Borcard \& Legendre, 2002)篎选显著的空间结构, 最后基于篮 选出的环境变量和PCNM特征向量进行变差分解, 以将物种组成变化分解为以下 3 个部分: (1)环境独 立解释量 $(E n v)$; (2) 空间独立解释量 $(G e o)$; (3)环境和 空间的联合解释量 $(E n v-G e o)$ 。

上述分析由 $\mathrm{R}$ 3.3.3软件完成, 样方间距离矩阵 计算、 $\beta$ 多样性计算以及RF分别由“fossil” (Vavrek, 2012) 、“ecodist” (Goslee et al., 2007) 和 “randomForest” (Ehrlinger, 2015)程序包实现, MRM 模型、db-RDA分析以及PCNM建模则由“vegan”程 序包实现(Dixon, 2003)。

\section{2 结果}

\section{1 地理距离和环境差异与群落构建的相关性}

回归分析结果显示, $\beta$ 多样性与地理距离与环境 差异均呈极显著的正相关关系 $(p<0.001$, 图2B), 表明 $\beta$ 多样性随地理距离和环境差异的增大而增大, 且 $\beta$ 多样性随地理距离的变化速率略大于其随环境 差异性的变化速率(地理距离 $r=0.518>$ 环境差异 $r=0.504$, 图2B)。这也就意味着地理距离和环境差 异越大, 样地间物种组成相似性越小。

\section{2 地理距离和环境因子对群落构建的解释量}

$\mathrm{RF}$ 和MRM模型的结果显示, 除乔木冠层高度 和草本层盖度外, 其余变量均对物种组成有显著影 响(图2A1、2A2)。对样地间物种组成变化解释率最 高的变量是地理距离, 其次为气候因子中的年降水 量、年平均气温以及生物因子中的胸高断面积和。 坡度、坡向以及乔木层密度的影响相对较小。

\section{3 地理距离和环境差异对群落构建的相对重要性}

根据变差分解结果, 全部环境因子和空间因子 共同解释了物种组成变化的 $47.8 \%$ (表1), 空间距离 和环境差异的独立解释率分别为 $14.1 \%$ 和 $9.8 \%$, 两 者联合解释率为 $23.9 \%$ 。地理距离的独立解释率略 

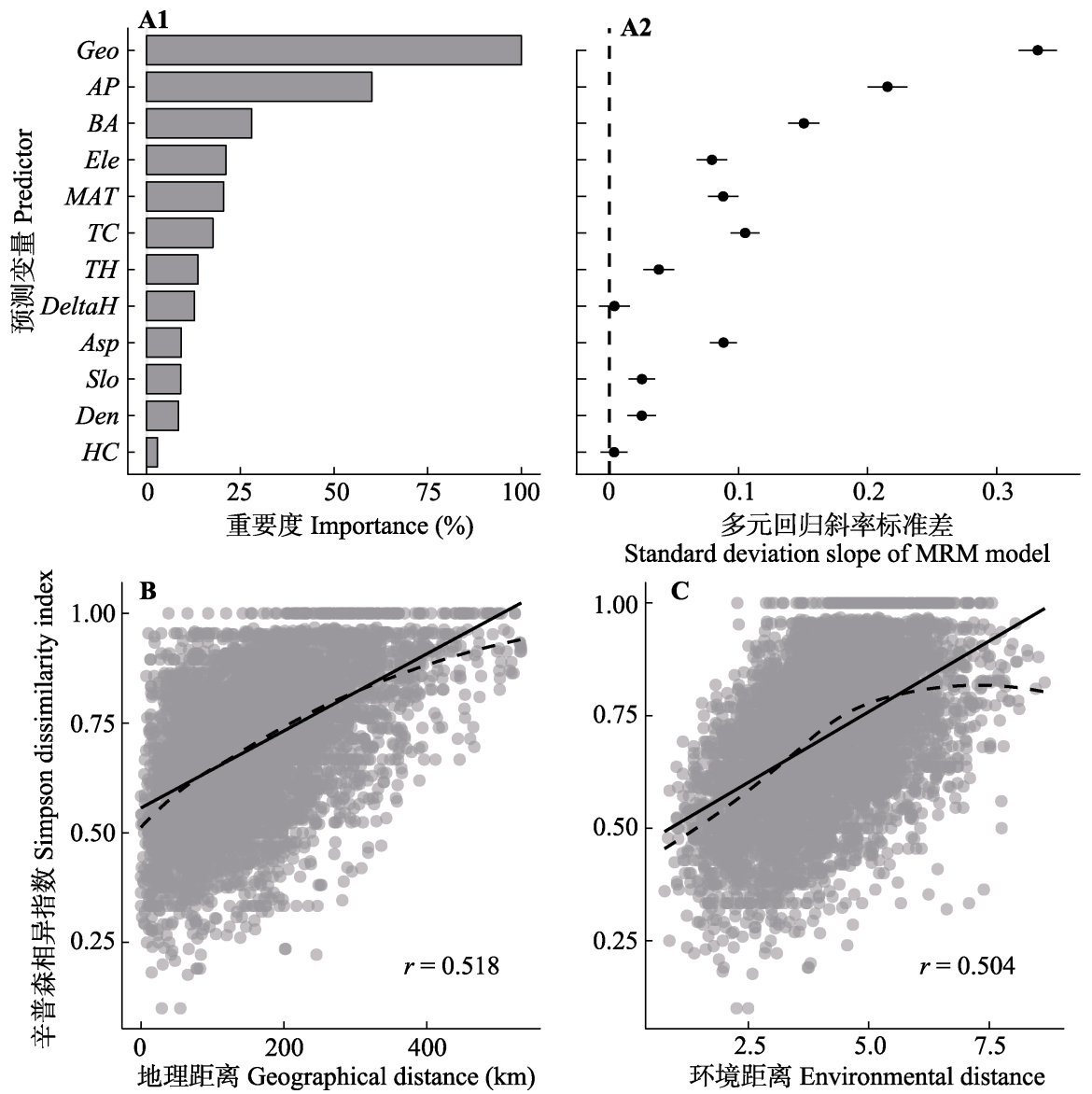

图2 随机森林模型 $(\mathrm{RF})$ 和基于距离矩阵的多元回归方法(MRM)结果。A1, A2, 分别为生态因子的重要性大小及显著性检验的 结果。 $\mathbf{B}, \beta$ 多样性与地理距离的关系。 $\mathbf{C}, \beta$ 多样性与环境距离的关系。 $A P$, 年降水量; $A s p$, 坡向; $B A$, 胸高断面积和; Den, 立 木密度; DeltaH, 冠层厚度; Ele, 海拔; Geo, 地理距离; HC, 草本层盖度; $M A T$, 年均气温; Slo, 坡度; TC, 乔木层盖度; $T H$, 乔 木高。虚线为Lowess拟合线, 实线为线性拟合回归线。

Fig. 2 Results of random forest (RF) model and multiple regression method (MRM) based on distance matrices. The importance of the ecological factors and the test results of significance are shown in $\mathbf{A 1}$ and $\mathbf{A 2}$, respectively; $\mathbf{B}$ and $\mathbf{C}$ respectively shows the relationship between dissimilarity of species composition with increasing geographical and environmental distance. $A P$, annual precipitation; Asp, aspect; $B A$, basal area; Den, density; DeltaH, delta height; Ele, elevation; Geo, geographical distance; $H C$, herbaceous coverage; MAT, mean annual temperature; Slo, slope; $T C$, tree coverage; $T H$, tree height. Dashed line represents Lowess line, solid for linear fitted regression line.

表1＼cjkstart黄土高原腹地人工林下草本群落物种组成变差分解结果(\%)

Table 1 Results of variation partitioning of understory species composition of the planted forests in the central Loess Plateau (\%)

\begin{tabular}{lc}
\hline 分解部分 Partitioned parts & 分解结果 \\
\hline 纯环境因子解释部分 Pure of each part \\
纯空间因子解释部分 Pure interpretation of environmental variables of geographic variables & 9.8 \\
联合解释部分 Joint interpretation & 14.1 \\
未解释部分 Unexplained part & 23.9 \\
\hline
\end{tabular}

大于环境差异的独立解释率, 两者的独立解释率则 均小于联合解释率，模型残差为 $52.2 \%$ 。

\section{3 讨论}

\section{$3.1 \beta$ 多样性与扩散距离的关系}

地理距离对林下草本层物种组成变化的独立解 释率为 $14.1 \%$, 这为中性理论在研究区的应用提供 了依据。随着地理距离的增加, 群落 $\beta$ 多样性表现为
明显的上升趋势(图2B)，表明扩散限制确实是黄土 高原地区林下草本群落构建过程中的限制因子之 一。Neil Gibson等(2017)对澳大利亚西南部沙地平 原的物种周转进行研究后也提出物种周转与地理距 离相关, 即认为扩散限制是影响物种周转的重要驱 动因子。一方面, 黄土高原沟壑纵横的地貌在一定 程度上阻碍了植物种子的传播(Saura \& PascualHortal, 2007; Urban et al., 2009), 但是扩散能力强的

www.plant-ecology.com 
种子能够扩散到更远的生境中, 这进一步体现扩散 限制对群落物种组成的影响。地理距离对研究区林 下草本群落 $\beta$ 多样性空间格局形成的重要性也可能 是因为扩散能力的差异性在一定程度上可以缓解种 间竞争(Mouquet \& Loreau, 2003), 从而改变群落的 物种组成。另一方面, 由于黄土高原环境恶劣, 生态 脆弱, 水热条件较差, 因而演替时间较短的人工林 下较难形成良好的枯枝落叶层，一些植物种子的传 播者无法在此生境中存活, 进而影响到依靠动物传 播的草本植物的扩散速率(Qian, 2009)。

\section{$3.2 \beta$ 多样性与环境因子的关系}

纯环境变量的独立解释率为 $9.8 \%$, 通过 RF和 $\mathrm{MRM}$ 分析, 进一步发现环境因子中年降水量与胸 高断面积和对物种组成变化的影响最大。首先, 研 究选取的人工林下草本群落位于半湿润-半干旱区 的黄土高原, 对于该区的草本物种而言, 水分是其 一大胁迫因子, 因此该区物种多样性通常与降水显 著相关(Wright et al., 1993)。其次, 研究区各样地间 年降水量差异较大。而由降水梯度驱动形成的物种 组成变化格局也已被证实, Toledo等(2011)在新热带 区(Neoptropics)的研究以及Réjou-Méchain等(2008) 在非洲热带森林的研究均表明群落物种组成受降水 梯度的影响。但不同于先前的研究, 气候因子中的 年平均气温在本区域中发挥的作用相对较小, 研究 对象不同可能是造成这一差异的主要原因。上述研 究多关注于木本植物, 而本研究则主要着眼于林下 草本植物。上层植被可以发挥缓冲作用, 如吸收一 定的太阳辐射和削弱风对林下草本层的影响(Liu et $a l ., 2015)$, 从而减少温度变化对林下物种组成的作 用。第三, 胸高断面积变量在一定程度上能够反映 林分的冠层结构特征(尤业明等, 2016), 而冠层结构 能够影响林分内土壤水分、光照环境以及温湿度等 微生境条件的变化(van Oijen et al., 2005), 这进一 步影响了林下草本物种组成。Ihaddaden等(2013)在 阿尔及利亚北部干旱区以及余敏等(2013)在山西小 蛇沟的研究也表明冠层结构对林下草本群落物种组 成具有重要影响。

\section{3 环境过滤和地理距离对群落构建的相对重要性}

$\beta$ 多样性随地理距离的衰减为中性过程在群落 物种组成过程中发挥的作用提供了证据, 而其与环 境距离的衰减关系也说明环境因素如年降水量、乔 木胸高断面积、年平均气温等也会影响群落的物种
组成。并且, 地理距离与环境因子的联合解释率大 于二者的独立解释率, 这表明生态位过程(确定性 过程)和中性过程(随机过程)共同作用于黄土高原区 林下草本层群落构建过程, 这与较多的前人研究结 果(卢品等, 2013; 谭珊珊等, 2013)相一致。

本研究结果显示, 相比于纯环境因子, 群落间 物种组成相似性变化更多地被空间因子所解释(表 1), 这可能是因为随机过程和确定过程的相对作用 大小具有尺度效应(Chase, 2014; Tan et al., 2017)。研 究尺度越大, 环境异质性越高, 环境因子对群落物 种组成的过滤作用越明显(Viana et al., 2016)。反之, 则随机过程的作用更为重要。如Jones等(2016)对澳 大利亚西南部植物区系、Tang等(2013)对中国三大 主要草原群落物种周转格局的大尺度研究结果均表 明环境过滤发挥了更为重要的作用。而Chust等 (2006)对巴拿马热带森林乔木 $\beta$ 多样性格局以及王 丹等(2013)对子午岭草地群落的小尺度研究则表明 地理距离在群落构建过程中占主导地位。与上述小 尺度研究相比, 虽然本研究区域南北跨度约达509 $\mathrm{km}$, 东西距离约为 $200 \mathrm{~km}$, 但可能由于黄土高原区 相对均质的气候和土壤等环境条件使得研究区仍然 没有包含足够的环境差异。因而即使研究区资源相 对贫乏, 水热条件较为严苛, 样地间的相似生境依 然减弱了环境过滤对物种周转的作用(Chase, 2014)。 与Bohlman等(2018)在亚马孙地区的研究得出的结 论相似。

从生活型的角度看, 与乔木相比, 林下草本植 物受气候, 尤其是受冬季低温的限制较小(Donoghue, 2008)。这可能是因为, 草本植物尤其是一年生 草本植物, 生活史周期较短, 这有利于其躲避极端 气候或干旱的胁迫。而当气候条件适宜时, 草本植 物也许可以从地下种库中迅速重建其种群(Grime, 1977)。因而, 水热条件对林下草本的限制作用相对 较小。另一方面, 草本植物倾向于产生质量较小, 利 于风媒传播的种子(赵鸣飞等, 2017), 这些策略均有 助于草本植物扩散到适宜其生存的生境中。理论上, 草本植物受扩散能力限制的可能性较小。但可能由 于黄土高原地区沟壑纵横, 破碎化程度较高, 特殊 的生境条件限制了该区林下草本植物的扩散距离。 除此之外, 某些环境因子(如年降水量、年平均气温) 的空间变化特征也可能会增加空间因子的解释率 
(Page \& Shanker, 2018)。

草本植物对气候等环境条件相对较低的依赖性 以及较强的扩散能力可能也解释了模型中环境因子 和空间因子对林下草本群落 $\beta$ 多样性的解释率较低 的原因。除此之外, 模型的较大残差 $(52.2 \%$, 表1) 还可能是受此次研究中尚未测量因子的影响。例如, 人为干扰以及土地利用方式等因子(Page \& Shanker, 2018); 再如, 土壤类型、土壤元素、土壤湿度等因 子也可能影响草本植物的定殖(Jones et al., 2008)。 但也有研究认为, 黄土高原的土壤类型较为单一, 土壤类型作为环境过滤的影响较小(王丹等, 2013)。

\section{4 结论}

研究通过揭示黄土高原人工林下草本层植物群 落 $\beta$ 多样性格局与空间距离和环境异质性之间的关 系, 为生态位理论和中性理论在群落构建过程中的 相对重要性提供了依据。研究表明, 以扩散限制为 代表的中性过程和以环境过滤为代表的生态位过程 对群落构建均起着重要作用。RF和MRM分析的结 果则进一步表明环境过滤主要通过年降水量与胸高 断面积和发挥作用。我们的模型解释了 $50 \%$ 左右的 物种组成变化, 未解释的部分可能是 $\beta$ 多样性还受 我们在此次研究中尚未考虑的人为干扰以及其他尚 未测量的环境和空间变量等因素的影响。另外, 不 可忽略的是环境因子具有空间自相关性, 因此可能 未被测量的生境因子增大了地理距离对本研究的解 释率, 这一问题将在以后的研究中作进一步的研究 证明。

致谢 感谢北京师范大学生命科学学院的杨容同 学、梁钰同学, 北京师范大学地理科学学部的李思 雨同学和北京师范大学政府管理学院的黄淮瑞同学 在野外工作和植物识别方面给予的帮助。

\section{参考文献}

Adler PB, HilleRisLambers J, Levine JM (2007). A niche for neutrality. Ecology Letters, 10, 95-104.

Anderson MJ, Crist TO, Chase JM, Vellend M, Inouye BD, Freestone AL, Sanders NJ, Cornell HV, Comita LS, Davies KF, Harrison SP, Kraft NJB, Stegen JC, Swenson NG (2011). Navigating the multiple meanings of $\beta$ diversity: A roadmap for the practicing ecologist. Ecology Letters, 14, 19-28.

Baselga A (2010). Partitioning the turnover and nestedness components of beta diversity. Global Ecology and Bio- geography, 19, 134-143.

Bohlman SA, Laurance WF, Laurance SG, Nascimento HEM, Fearnside PM, Andrade A (2008). Importance of soils, topography and geographic distance in structuring central Amazonian tree communities. Journal of Vegetation Science, 19, 863-874.

Borcard D, Legendre P (2002). All-scale spatial analysis of ecological data by means of principal coordinates of neighbour matrices. Ecological Modelling, 153, 51-68.

Breiman L (2001). Random forests. Machine Learning, 45, 5-32.

Burrascano S, Sabatini FM, Blasi C (2011). Testing indicators of sustainable forest management on understorey composition and diversity in southern Italy through variation partitioning. Plant Ecology, 212, 829-841.

Chase JM (2014). Spatial scale resolves the niche versus neutral theory debate. Journal of Vegetation Science, 25, 319-322.

Chase JM, Myers JA (2011). Disentangling the importance of ecological niches from stochastic processes across scales. Philosophical Transactions of the Royal Society of London, 366, 2351-2363.

Chust G, Chave J, Condit R, Aguilar S, Lao S, Pérez R (2006). Determinants and spatial modeling of tree $\beta$-diversity in a tropical forest landscape in Panama. Journal of Vegetation Science, 17, 83-92.

Condit R, Hubbell SP (2002). Beta-diversity in tropical forest trees. Science, 295, 666-669.

Condit R, Pitman N, Leigh Jr EG, Chave J, Terborgh J, Foster RB, Núñez V. P, Aguilar S, Valencia R, Villa G, Muller-Landau HC, Losos E, Hubbell SP (2002). Betadiversity in tropical forest trees. Science, 295, 666-669.

Dixon P (2003). VEGAN, a package of R functions for community ecology. Journal of Vegetation Science, 14, 927-930.

Donoghue MJ (2008). A phylogenetic perspective on the distribution of plant diversity. Proceedings of the National Academy of Sciences of the United States of America, 105, 11549-11555.

Ehrlinger J (2015). ggRandomForests: Visually Exploring a Random Forests. https://cran.r-project.org/web/packages/ ggRandomForests. Cited: 2018-07-03

Fu BJ, Liu Y, Lü Y, He CS, Zeng Y, Wu BF (2011). Assessing the soil erosion control service of ecosystems change in the Loess Plateau of China. Ecological Complexity, 8, 284-293.

George LO, Bazzaz FA (2014). The herbaceous layer as a filter determining spatial pattern in forest tree regeneration. In: Gilliam FS, Roberts MR eds. The Herbaceous Layer in Forests of Eastern North America. Oxford University Press, New York.

Gibson N, Prober S, Meissner R, Van Leeuwen S (2017). Implications of high species turnover on the south-western Australian sandplains. PLOS ONE, 12, e0172977. DOI: 10.1371/journal.pone.0172977.

Gilliam FS (2007). The ecological significance of the herbaceous layer in temperate forest ecosystems. Bioscience, 57,

www.plant-ecology.com 
$845-858$.

Gilliam FS, Hockenberry AW, Adams MB (2006). Effects of atmospheric nitrogen deposition on the herbaceous layer of a Central Appalachian hardwood forest. Journal of the Torrey Botanical Society, 133, 240-254.

Goslee SC, Urban DL, Leeuw JD, Zeileis A (2007). The ecodist package for dissimilarity-based analysis of ecological data. Journal of Statistical Software, 22, 1-19.

Gravel D, Canham CD, Beaudet M, Messier C (2006). Reconciling niche and neutrality: The continuum hypothesis. Ecology Letters, 9, 399-409.

Grime JP (1977). Evidence for the existence of three primary strategies in plants and its relevance to ecological and evolutionary theory. The American Naturalist, 111, 1169-1194.

Hubbell SP (2001). The Unified Neutral Theory of Biodiversity and Biogeography. Princeton University Press, Princeton, USA.

Ihaddaden A, Velázquez E, Rey-Benayas JM, Kadi-Hanifi H (2013). Climate and vegetation structure determine plant diversity in Quercus ilex woodlands along an aridity and human-use gradient in Northern Algeria. Flora, 208, 268-284.

Jamoneau A, Passy SI, Soininen J, Leboucher T, TisonRosebery J (2018). Beta diversity of diatom species and ecological guilds: Response to environmental and spatial mechanisms along the stream watercourse. Freshwater Biology, 63, 62-73.

Jones MM, Gibson N, Yates C, Ferrier S, Mokany K, Williams KJ, Manion G, Svenning JC (2016). Underestimated effects of climate on plant species turnover in the Southwest Australian Floristic Region. Journal of Biogeography, 43, 289-300.

Jones MM, Tuomisto H, Borcard D, Legendre P, Clark DB, Olivas PC (2008). Explaining variation in tropical plant community composition: Influence of environmental and spatial data quality. Oecologia, 155, 593-604.

Kraft NJB, Comita LS, Chase JM, Sanders NJ, Swenson NG, Crist TO, Stegen JC, Vellend M, Boyle B, Anderson MJ, Cornell HV, Davies KF, Freestone AL, Inouye BD, Harrison SP, Myers JA (2011). Disentangling the drivers of $\beta$ diversity along latitudinal and elevational gradients. Science, 333, 1755-1758.

Legendre P, Anderson MJ (1999). Distance-based redundancy analysis: Testing multispecies responses in multifactorial ecological experiments. Ecological Monographs, 69, 1-24.

Legendre P, de Cáceres M (2013). Beta diversity as the variance of community data: Dissimilarity coefficients and partitioning. Ecology Letters, 16, 951-963.

Legendre P, Lapointe FJ, Casgrain P (1994). Modeling brain evolution from behavior: A permutational regression approach. Evolution, 48, 1487-1499.

Legendre P, Mi XC, Ren HB, Ma KP, Yu MJ, Sun IF, He FL (2009). Partitioning beta diversity in a subtropical broadleaved forest of China. Ecology, 90, 663-674.

Leibold MA, McPeek MA (2006). Coexistence of the niche and neutral perspectives in community ecology. Ecology, 87, 1399-1410.

Liu HY, Yin Y, Wang QY, He SY (2015). Climatic effects on plant species distribution within the forest-steppe ecotone in northern China. Applied Vegetation Science, 18, 43-49.

Liu QF, Kang MY, Liu QR (2006). Quantitative classification and environmental interpretation of forest tree species in Hungou, Zhongtiao Mountain. Journal of Plant Ecology (Chinese Version), 30, 383-391. [刘秋锋, 康慕谊, 刘全 儒 (2006). 中条山混沟地区森林乔木种的数量分类与 环境解释. 植物生态学报, 30, 383-391.]

Lu P, Jin Y, Chen JH, Li MH, Yu MJ (2013). Influence of geographical distance and topographic difference on $\beta$ diversity in two large-scale forest dynamics plots. Biodiversity Science, 21，554-563. [卢品, 金毅, 陈建华, 李铭红, 于 明坚 (2013). 地理距离和地形差异对两个大型森林动 态样地 $\beta$ 多样性的影响. 生物多样性, 21, 554-563.]

Márialigeti S, Tinya F, Bidló A, Ódor P (2016). Environmental drivers of the composition and diversity of the herb layer in mixed temperate forests in Hungary. Plant Ecology, 217, 549-563.

Marmion M, Luoto M, Heikkinen RK, Thuiller W (2009). The performance of state-of-the-art modelling techniques depends on geographical distribution of species. Ecological Modelling, 220, 3512-3520.

May F, Huth A, Wiegand T (2015). Moving beyond abundance distributions: Neutral theory and spatial patterns in a tropical forest. Proceedings of the Royal Society B Biological Sciences, 282, 20141657. DOI: 10.1098/rspb.2014.1657.

Mouquet N, Loreau M (2003). Community patterns in sourcesink metacommunities. The American Naturalist, 162, 544-557.

Myers JA, Chase JM, Jiménez I, Jørgensen PM, AraujoMurakami A, Paniagua-Zambrana N, Seidel R (2013). Beta-diversity in temperate and tropical forests reflects dissimilar mechanisms of community assembly. Ecology Letters, 16, 151-157.

Page NV, Shanker K (2018). Environment and dispersal influence changes in species composition at different scales in woody plants of the Western Ghats, India. Journal of Vegetation Science, 29, 74-83.

Petermann JS, Kratina P, Marino NAC, MacDonald AAM, Srivastava DS (2015). Resources alter the structure and increase stochasticity in bromeliad microfauna communities. PLOS ONE, 10, e0118952. DOI: 10.1371/journal. pone.0118952.

Qian H (2009). Beta diversity in relation to dispersal ability for vascular plants in North America. Global Ecology and Biogeography, 18, 327-332.

Réjou-Méchain M, Pélissier R, Gourlet-Fleury S, Couteron P, Nasi R, Thompson JD (2008). Regional Variation in tropical forest tree species composition in the Central African Republic: An assessment based on inventories by forest companies. Journal of Tropical Ecology, 24, 663-674.

Sabatini F, Jiménez-Alfaro B, Burrascano S, Blasi C (2014). 
Drivers of herb-layer species diversity in two unmanaged temperate forests in northern Spain. Community Ecology, $15,147-157$.

Saura S, Pascual-Hortal L (2007). A new habitat availability index to integrate connectivity in landscape conservation planning: Comparison with existing indices and application to a case study. Landscape and Urban Planning, 83, 91-103.

Seidler TG, Plotkin JB (2006). Seed dispersal and spatial pattern in tropical trees. PLOS Biology, 4, e344. DOI: 10.1371/journal.pbio.0040344.

Siefert A, Ravenscroft C, Weiser MD, Swenson NG (2013). Functional beta-diversity patterns reveal deterministic community assembly processes in eastern North American trees. Global Ecology and Biogeography, 22, 682-691.

Smith TW, Lundholm JT (2010). Variation partitioning as a tool to distinguish between niche and neutral processes. Ecography, 33, 648-655.

Sun L, Zhang GH, Luan LL, Li ZW, Geng R (2016). Distribution of soil organic carbon in surface soil along a precipitation gradient in loess hilly. Chinese Journal of Applied Ecology, 27, 532-538. [孙龙, 张光辉, 奕莉莉, 李振炜, 耿韧 (2016). 黄土丘陵区表层土壤有机碳沿降 水梯度的分布. 应用生态学报, 27, 532-538.]

Tan LZ, Fan CY, Zhang CY, von Gadow K, Fan XH (2017). How beta diversity and the underlying causes vary with sampling scales in the Changbai mountain forests. Ecology and Evolution, 7, 10116-10123.

Tan SS, Ye ZL, Yuan LB, Zhou RF, Hu G, Jin XF, Yu MJ (2013). Beta diversity of plant communities in Baishanzu Nature Reserve. Acta Ecologica Sinica, 33, 6944-6956. [谭珊珊, 叶珍林, 袁留斌, 周荣飞, 胡广, 金孝锋, 于 明坚 (2013). 百山祖自然保护区植物群落beta多样性. 生态学报, 33, 6944-6956.]

Tang ZY, Fang JY, Chi XL, Yang YH, Ma WH, Mohhamot A, Guo ZD, Liu YN, Gaston KJ (2012). Geography, environment, and spatial turnover of species in China's grasslands. Ecography, 35, 1103-1109.

Toledo M, Poorter L, Peña-Claros M, Alarcón A, Balcázar J, Chuviña J, Leaño C, Licona JC, ter Steege H, Bongers F (2011). Patterns and determinants of floristic variation across lowland forests of Bolivia. Biotropica, 43, 405-413.

Tuomisto H, Ruokolainen K, Vormisto J, Duque A, Sánchez M, Paredes VV, Lähteenoja O (2017). Effect of sampling grain on patterns of species richness and turnover in Amazonian forests. Ecography, 40, 840-852.

Urban DL, Minor ES, Treml EA, Schick RS (2009). Graph models of habitat mosaics. Ecology Letters, 12, 260-273.

van Oijen D, Feijen M, Hommel P, den Ouden J, de Waal R (2005). Effects of tree species composition on within-forest distribution of understorey species. Applied Vegetation Science, 8, 155-166.

Vavrek MJ (2012). Fossil: Palaeoecological and palaeogeographical analysis tools. Palaeontologia Electronica,
$14,50-64$.

Viana DS, Figuerola J, Schwenk K, Manca M, Hobæk A, Mjelde M, Preston CD, Gornall RJ, Croft JM, King RA, Green AJ, Santamaría L (2016). Assembly mechanisms determining high species turnover in aquatic communities over regional and continental scales. Ecography, 39, 281-288.

Wang D, Wang XA, Guo H, Wang SX, Zhen WN, Liu SL (2013). Effect of species dispersal and environmental factors on species assemblages in grassland communities. Acta Ecologica Sinica, 33，4409-4415. [王丹，王孝安， 郭华, 王世雄, 郑维娜, 刘史力 (2013). 环境和扩散对 草地群落构建的影响. 生态学报, 33, 4409-4415.]

Wang SX, Zhao L, Li N, Guo H, Wang XA, Duan RY (2016). Community heterogeneity of undergrowth vegetation in Pinus tabuliformis forest on the Loess Plateau of Northwest China. Chinese Journal of Ecology, 35, 1197-1203. [王世雄, 赵亮, 李娜, 郭华, 王孝安, 段仁燕 (2016). 黄土高原油松林林下群落异质性分析. 生态学杂志, 35, 1197-1203.]

Wright DH, Currie DJ, Maurer BA (1993). Energy supply and patterns of species richness on local and regional scales. In: Ricklef RE, Schluter D eds. Species Diversity in Ecological Communities: Historical and Geographical Perspectives. University of Chicago Press, Chicago. 66-74.

Xiao JF (2014). Satellite evidence for significant biophysical consequences of the "Grain for Green" Program on the Loess Plateau in China. Journal of Geophysical Research Biogeosciences, 119, 2261-2275.

You YM, Xu JY, Cai DX, Liu SR, Zhu HG, Wen YG (2016). Environmental factors affecting plant species diversity of understory plant communities in a Castanopsis hystrix plantation chronosequence in Pingxiang, Guangxi, China. Acta Ecologica Sinica, 36, 164-172. [尤业明, 徐佳玉, 蔡道雄, 刘世荣, 朱宏光, 温远光 (2016). 广西凭祥不 同年龄红椎林林下植物物种多样性及其环境解释. 生 态学报, 36, 164-172.]

Yu M, Zhou ZY, Kang FF, Ouyang S, Mi XC, Sun JX (2013). Gradient analysis and environmental interpretation of understory herb-layer communities in Xiaoshegou of Lingkong Mountain, Shanxi, China. Chinese Journal of Plant Ecology, 37, 373-383. [余敏, 周志勇, 康峰峰, 欧 阳帅, 米湘成, 孙建新 (2013). 山西灵空山小蛇沟林下 草本层植物群落梯度分析及环境解释. 植物生态学报, 37, 373-383.]

Zhao MF, Wang GY, Xing KX, Wang YH, Xue F, Kang MY, Luo K (2017). Patterns and determinants of species similarity decay of forest communities in the western Qinling Mountains. Biodiversity Science, 25, 3-10. [赵鸣 飞, 王国义, 邢开雄, 王宇航, 薛峰, 康慕谊, 罗开 (2017). 秦岭西部森林群落相似性递减格局及其影响因 素. 生物多样性, 25, 3-10.]

责任编委: 唐志尧 责任编辑: 李 敏 实习编辑: 赵 航 